$\mathrm{AD}$

Award Number:

W81XWH-07-1-0325

TITLE :

Multiplex Quantitative Histologic Analysis of Human Breast Cancer Cell Signaling and Cell Fate

PRINCIPAL INVESTIGATOR:

William M. F. Lee

Badrinath Roysam

CONTRACTING ORGANIZATION:

University of Pennsylvania

Philadelphia, PA 19104

REPORT DATE :

May 2008

TYPE OF REPORT:

Annual

PREPARED FOR: U.S. Army Medical Research and Materiel Command Fort Detrick, Maryland 21702-5012

DISTRIBUTION STATEMENT:

Approved for public release; distribution unlimited

The views, opinions and/or findings contained in this report are those of the author(s) and should not be construed as an official Department of the Army position, policy or decision unless so designated by other documentation. 


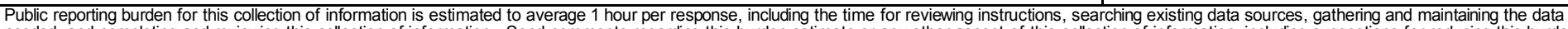

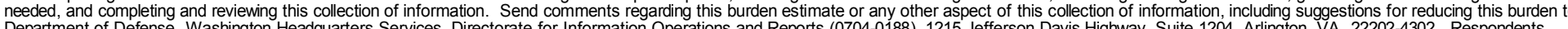

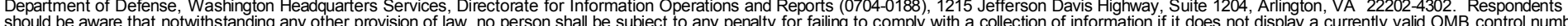
should be awa that notwithstanding any other provision of law, no person shal

1. REPORT DATE (DD-MM-YYYY)

2. REPORT TYPE

Annual

$01 / 05 / 2008$

4. TITLE AND SUBTITLE

Multiplex Quantitative Histologic Analysis of Human Breast Cancer Cell Signaling

And Cell Fate

6. AUTHOR(S)

William Lee

Badrinath Roysam

7. PERFORMING ORGANIZATION NAME(S) AND ADDRESS(ES)

University of Pennsylvania

Philadelphia, PA 19104

9. SPONSORING / MONITORING AGENCY NAME(S) AND ADDRESS(ES)

10. SPONSOR/MONITOR'S ACRONYM(S)

U.S. Army Medical Research and Materiel Command

Fort Detrick, MD 21702-5012

1. SPONSOR/MONITOR'S REPORT NUMBER(S)

12. DISTRIBUTION / AVAILABILITY STATEMENT

Approved for public release. distribution unlimited

\section{SUPPLEMENTARY NOTES}

\section{ABSTRACT}

Many molecular events and cellular processes are preserved in fixed human tumor specimens, and access to this wealth of information about human cancers in authentic context awaits a method for them to be quantified and analyzed. Some, such as cell signaling and cell fate decisions, are prognostically and therapeutically important, and can be revealed by immunohistological staining. We are developing a novel platform for immunohistological study of breast cancer specimens that will retrieve multiplex quantitative molecular information about tumor cells at a cytologic level. The platform will use multispectral microscopy to examine breast cancer specimens that have been immunostained for multiple structural and functional antigens using different chromogens and fluorophores. Staining for structural antigens (nuclei, epithelial cytokeratins, E-cadherin), allows cells to be identified and classified as breast cancer cells. Staining for cell signaling and fate antigens (p-ERK, p-AKT, Ki-67) reports on these important biological processes and events in cells. Multispectral microscopy permits staining for individual antigens to be distinguished and separated from staining for other antigens in multiplex-stained slides. The other component of the proposed platform is software for analyzing multispectral images individually and associatively. Algorithms will identify and classify cells in images, attribute signaling and cell fate events and processes to each cell, and reveal relationships among events through analysis of association among their stains.

15. SUBJECT TERMS

Breast cancer, cell signaling, cell proliferation, histology, image analysis

\begin{tabular}{|c|c|c|c|c|c|}
\hline \multicolumn{3}{|c|}{ 16. SECURITY CLASSIFICATION OF: } & \multirow{2}{*}{$\begin{array}{l}\text { 17. LIMITATION } \\
\text { OF ABSTRACT } \\
\text { unlimited }\end{array}$} & \multirow{2}{*}{$\begin{array}{l}\text { 18. NUMBER } \\
\text { OF PAGES } \\
7\end{array}$} & \multirow{2}{*}{$\begin{array}{l}\text { 19a. NAME OF RESPONSIBLE PERSON } \\
\text { USAMRMC } \\
\text { 19b. TELEPHONE NUMBER (include area } \\
\text { code) }\end{array}$} \\
\hline $\begin{array}{l}\text { a. REPORT } \\
\text { unclassified }\end{array}$ & $\begin{array}{l}\text { b. ABSTRACT } \\
\text { unclassified }\end{array}$ & $\begin{array}{l}\text { c. THIS PAGE } \\
\text { unclassified }\end{array}$ & & & \\
\hline
\end{tabular}




\section{Table of Contents}

Page

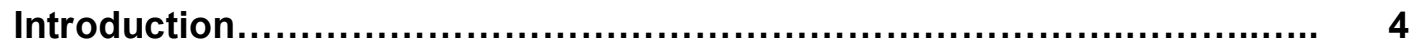

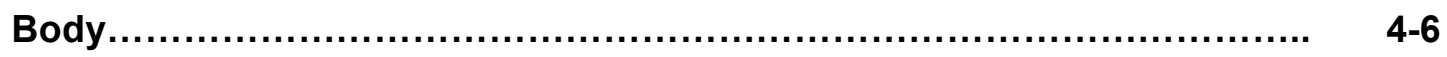

Key Research Accomplishments...................................... $\quad 6-7$

Reportable Outcomes........................................................ 7

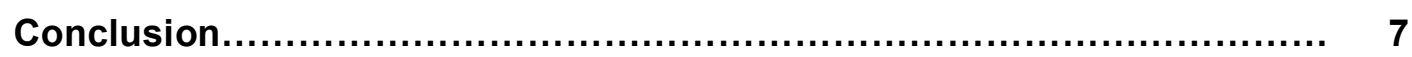

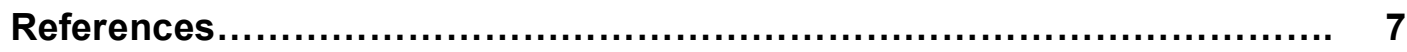

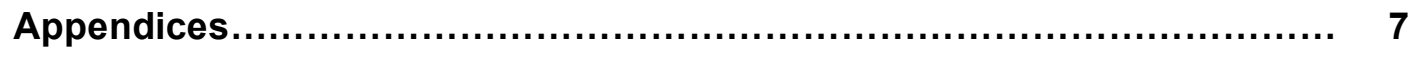




\section{Principal Investigator: $\quad$ Lee, William M. F. \\ Proposal Title: \\ Multiplex Quantitative Histologic Analysis of Human Breast Cancer Cell Signaling and Cell Fate}

Introduction:

The aim of this proposal is to build an advanced platform for immunohistological study of breast cancer specimens that will retrieve multiplex quantitative molecular information on a cellular basis. Three components will be developed to generate this platform. (1) Tissue staining protocols for revealing multiple structural and functional parameters plus conventional histology in the same histologic section. (2) Imaging by multispectral microscopy (Nuance, CRi, Woburn, MA) to capture the information revealed by individual stains in multiplex combinations. (3) Software for automated multispectral data analysis that classifies cells in images based on structural antigen staining or spatial and textural analysis, assigns processes and events to cells based on staining for functional antigens, and determines event relationships by association of their stains at a cellular level. Operation of the platform and the quantitative information it provides will be tested using breast tumors of various histologic subtypes, including pre- and post-therapy tumor biopsies from patients treated with an EGFR/HER2 inhibitor.

\section{Body of Report:}

Task 1. Develop protocols for multiplex staining of human breast cancer specimens for antigens reporting cell signaling events and cell fate decisions

We developed immunostaining protocols that sensitively detect antigens in paraffin-embedded human breast cancer specimens that report on cell signaling (p-ERK, p-AKT) and cell fate decisions (Ki-67, TUNEL). Immunohistochemical (IHC) staining for these analytes using chromogenic substrates (e.g. $\mathrm{DAB}$, diaminobenzidine) yielded the highest percentage of positive tumor cells, although immunofluorescent (IF) staining using fluorescent reporters yielded comparable percentages of p-ERK and Ki-67 positive cells. This indicated that immunostaining using IF or IHC reporters were similarly sensitive of detecting p-ERK and Ki-67. For p-AKT, IF staining yielded far fewer $(<25 \%)$ positive cells, indicating that IF was much less sensitive than IHC for p-AKT detection. With the antibodies currently available, we consider p-ERK and Ki67 immunostaining to be quantifiable by IF or IHC, but p-AKT is quantifiable only by IHC. Because of the greater sensitivity of immunostaining by IHC, we have adopted this approach for revealing all our analytes. Because chromogens are being used to reveal analytes, we use hematoxylin chromogenic staining and not DAPI fluorescent staining to reveal nuclei (strong nuclear chromogenic analyte staining prevents detection of DAPI fluorescence, and these DAPI-stained nuclei are often not counted during subsequent image analysis).

To study two analytes in the same tumor section or slide (e.g. p-ERK + Ki67 or p-AKT + Ki67), we developed dual analyte immunostaining protocols that provided quantitation for each antigen similar to that provided by staining for only a single antigen. For accurate quantitation of both analytes during dual analyte immunostaining, both antigens had to be stained by IHC or both had to be stained by IF. With dual IHC immunostaining, one of the two antigens is stained using DAB and the other is stained using another chromogen (e.g. SG Blue), and Nuance multispectral microscopy is used to detect and separate staining by the two chromogens, even when they are co-localized. The problem with combining IHC and IF reporters in dual analyte immunostaining is that DAB quenches the fluorescent reporter, and quantitation of the antigen reported by IF staining is compromised.

Next, we develop protocols for combining immunostaining for analytes (p-ERK, p-AKT, Ki67) and for epithelial cytokeratin (CK), so that tumor cells may be identified during subsequent automated image analysis (as CK+ cells). CK could be easily detected by single-step IF staining using anti-CK antibody that had been fluorescently labeled in vitro, which simplifies and speeds up the immunostaining protocol. IF immunostaining for CK can be used with IHC immunostaining for p-ERK, p-AKT or Ki67, because (a) CK does not need to be quantitated and (b) it is a cytoplasmic antigen and does not 
overlap with analyte immunostaining, which is predominantly or exclusively nuclear (DAB quenches fluorescence only when they are co-localized). We have also develop protocols for combining immunostaining for analytes and for E cadherin (ECad), so that tumor cell plasma membrane boundaries may be delineated during subsequent automated image analysis. We are in the process of determining specimen handling conditions under which p-ERK, p-AKT and Ki67 epitopes are preserved.

\section{Task 2. Optimize multispectral imaging and data capture for subsequent computational analysis}

We have tested various image capture conditions and parameters to determine the optimal parameters for acquiring images for accurate analyte quantification and adequate sampling of breast cancer specimens. We usually perform analysis at 400X magnification and acquire 150-400 breast cancer cells for analysis from each image. We acquire 4-10 images from each specimen, so that 1500-3000 tumor cells are analyzed. The Nuance multispectral imager has no difficulty separating hematoxylin, DAB and SG Blue chromogens when they are in the same space. It also has no difficulty separating ECad and CK when these are immunostained with red and green fluorescent reporters, respectively.

\section{Task 3. Develop computational algorithms for multispectral immunohistological image analysis}

A software tool to quantify intrinsic and associative parameters associated with whole cell segmentation from multi-spectral image data recorded at the PI's laboratory at U. Penn has been developed. The system has been implemented using the IDL software development platform (ITT Industries), and is currently undergoing evaluation at the PI's laboratory.

The first step in the image analysis is automatic segmentation of all cell nuclei based on hematoxylin staining of nuclei (Figure 1). In this regard, we have developed a novel algorithm based on multi-scale image analysis to locate the "marker points" shown as green dots in Figure 1 that indicate centers of putative nuclei. The use of these markers greatly simplifies the task of inspecting and editing nucler segmentation results since the user only needs to add or remove markers. We have found this to be efficient and effective. Once the user approves the marker results, the system automatically delineates the nuclei using a novel graph-cuts segmentation algorithm that significantly improves upon our prior work.

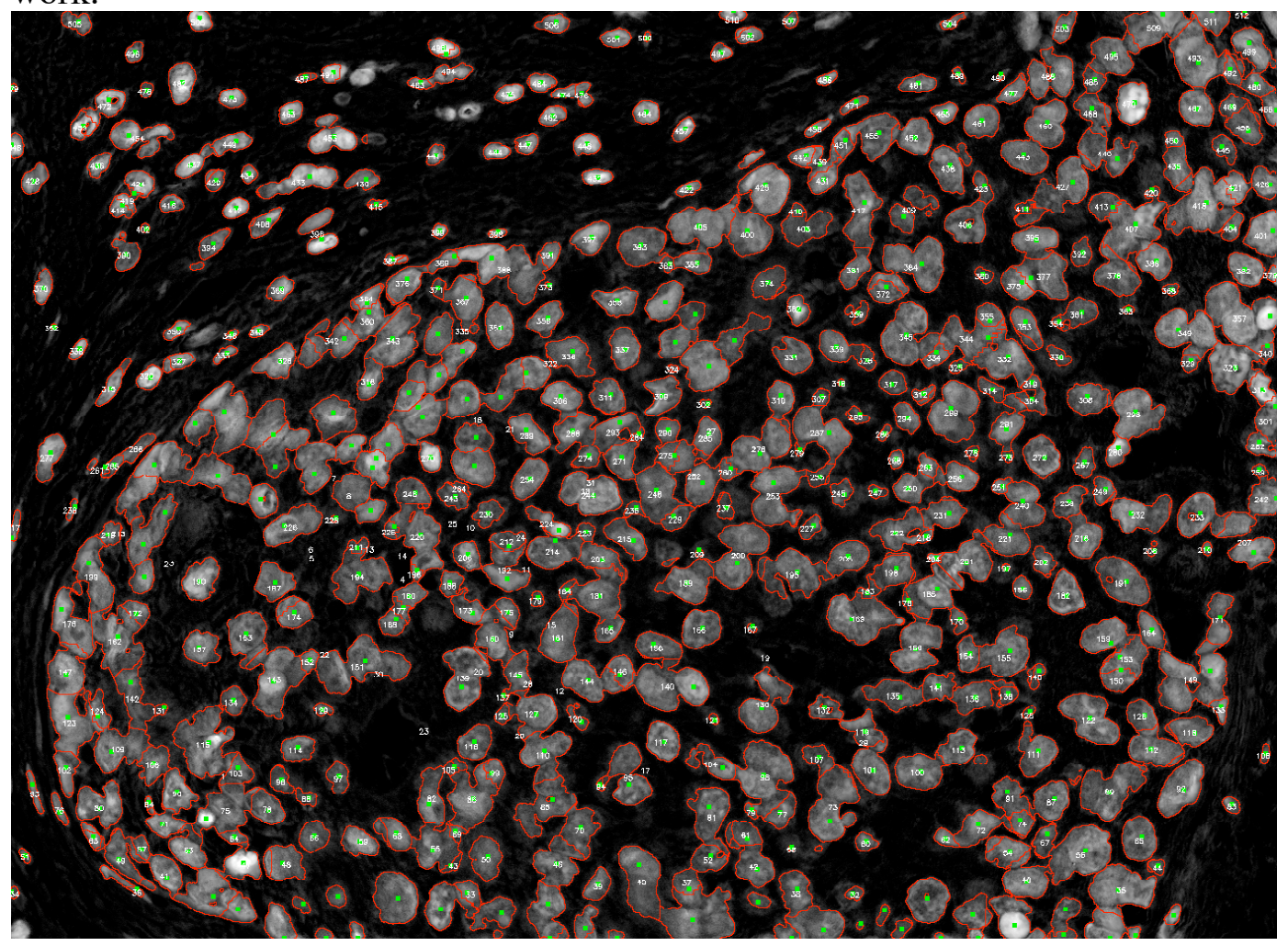


The second step is automated delineation of cellular domains from a cell membrane bound marker (E cadherin), as shown in Figure 2 below. This involves: computing the principal eigenvalue of Hessian of the membrane image; grayscale thinning to obtain 1-pixel thick membrane boundaries from the principal eigenvalue image. These segmentations have the valuable property of always resulting in closed contours for the cells even when there are unavoidable discontinuities in the ECad signal. This result too can be inspected and edited by the user.

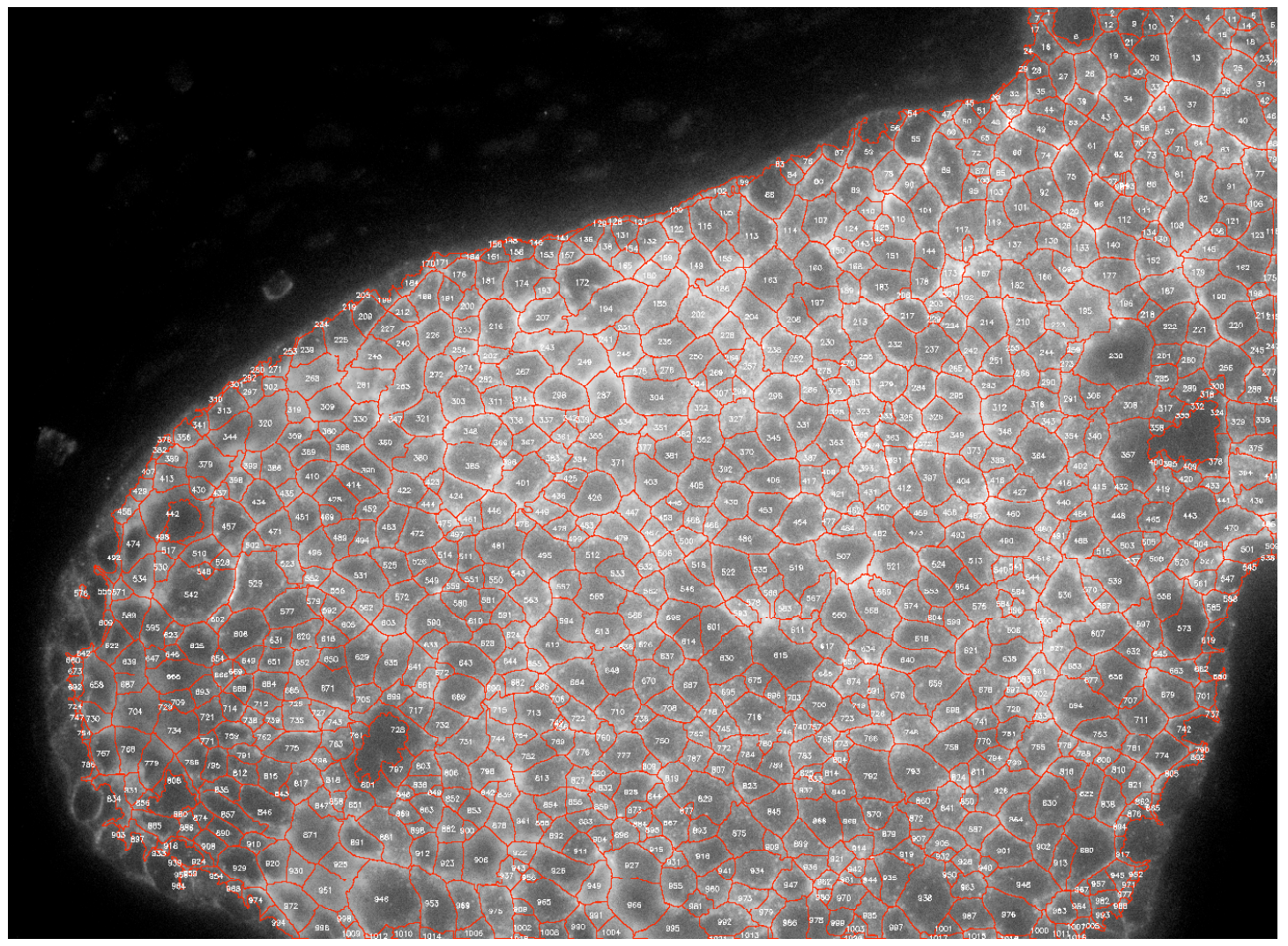

Once this is done, the software system quantifies the amount of fluorescence signal associated with multiple analytes. Specifically, it computes the amount of cytokeratin (CK) signal in each cell as a feature to differentiate tumor cells from non-tumor cells.

\section{Task 4. Validate immunohistological analysis system and data obtained}

This task awaits completion of task 3, which will provide an analytical software package ready for testing on clinical samples.

\section{Key Research Accomplishments}

We have developed robust tissue immunostaining protocols that allow breast cancer specimens to be stained for combinations of p-ERK, p-AKT and Ki-67 analyte using chromogens along with histochemical staining for nuclei (with hematoxylin) and immunostaining for cytosol (CK) and plasma membrance (ECad) by fluorescence.

We have optimized multispectral image capture of specimens immunostained for multiple antigens and structures such that staining for each is separated into distinct channels. 
We have optimized software for nuclear segmentation based on hematoxylin staining.

We have begun developing software for whole cell segmentation that divides each cell in the image into nuclear, cytosolic and plasma membrane compartments.

\section{Reportable Outcomes (Publication bibliography)}

No publications yet.

Personnel receiving pay: William Lee (PI, University of Pennsylvania, Philadelphia, PA) Badrinath Roysam (co-PI, Rensselaer Polytechnic Institute, Troy, NY) Sumit Nath (postdoc, Rensselaer Polytechnic Institute, Troy, NY)) Wiem Lassoued (grad student/research specialist, University of Pennsylvania, Philadelphia, PA)

\section{Conclusions}

Multiple antigens reporting on cell signaling and cell fate decisions can be stained for in human breast cancer specimens along with markers of different subcellular compartments in tumor cells.

These antigens and subcellular compartment markers can be separated into separate channels using multispectral microscopy for subsequent image analysis.

Accurate and rapid nuclear segmentation and nuclear analyte association can be performed computationally.

\section{References and Appendices}

None 